\title{
The management of chronic osteomyelitis in adults: outcomes of an integrated approach
}

\author{
Rudolph G Venter ${ }^{(D)}$, Yashwant S Tanwar $\left.{ }^{(}\right)$, Jan-Petrus Grey $(\mathbb{D}$, Nando Ferreira*(i) \\ Division of Orthopaedic Surgery, Department of Surgical Sciences, Faculty of Medicine and Health Sciences, Tygerberg Hospital, Stellenbosch University,
} Cape Town, South Africa

*Corresponding author: nferreira@sun.ac.za

Citation: Venter RG, Tanwar

YS, Grey JP, Ferreira N.

The management of chronic osteomyelitis in adults: outcomes

of an integrated approach. SA

Orthop J 2021;20(1):33-38.

http://dx.doi.org/10.17159/2309-

8309/2021/v20n1a4

Editor: Dr Franz Birkholtz, Walk-A-Mile Centre for Advanced Orthopaedics, Pretoria, South Africa

Received: July 2020

Accepted: September 2020

Published: March 2021

Copyright: @ 2021 Venter $\mathrm{RG}$. This is an open-access article distributed under the terms of the Creative Commons Attribution Licence, which permits unrestricted use, distribution and reproduction in any medium, provided the original author and source are credited.

Funding: No funding was received for this manuscript.

Conflict of interest: All the authors declare they have no conflicts of interest that are directly or indirectly related to the research.

\section{Abstract \\ Background}

This study presents the outcomes of the management of chronic osteomyelitis of the appendicular skeleton according to an integrated approach at a dedicated bone infection unit in South Africa.

\section{Methods}

A retrospective record review identified 80 patients who were treated between January 2016 and December 2018.

\section{Results}

Sixty patients (75\%) presented with fracture-related infections, 17 patients $(21 \%)$ developed chronic osteomyelitis following haematogenous spread and three (4\%) from contiguous wounds. According to the Cierny and Mader classification, 21 patients presented with anatomical type I, 14 with type II, 24 with type III and 21 with type IV chronic osteomyelitis. Positive microbial cultures were obtained in $63(79 \%)$ cases. Follow-up for the cohort ranged from 1 to 29 months, with a mean follow-up of 10.4 months. The overall complication rate for the cohort was $6 \%$ and included sterile drainage from the surgical site after management with bioactive glass (S53P4), refracture after hardware removal, and development of non-union. Five patients experienced recurrence after the initial procedure to eradicate infection, resulting in an overall resolution rate of $94 \%$.

\section{Conclusion}

Using single-stage surgeries and tailored dead space management strategies according to a comprehensive integrated approach developed in South Africa, results comparable to international literature can be achieved.

\section{Level of evidence: Level 4}

Keywords: chronic osteomyelitis, dead space management, bioactive glass, Lautenbach technique

\section{Introduction}

Chronic osteomyelitis is notoriously difficult to eradicate, and high failure rates have been reported in the literature. ${ }^{1,2}$ The fact that certain bacteria form biofilm with metabolically inactive colonies and can become dormant intracellularly in osteocytes, contributes to the difficulty in achieving cure in these cases. ${ }^{3-8}$ Although no evidence-based treatment guidelines exist for the management of chronic osteomyelitis, the ideal treatment strategy can be outlined as: resection of all necrotic tissue, dead space management, followed by soft tissue and bony reconstruction as required., .9-12 $^{3}$ Concurrent antibiotic therapy is initiated as an adjunct to surgical management. ${ }^{12}$

The most widely used classification system is that of Cierny and Mader, incorporating both the local extent of the disease, and the physiological status of the patient to guide management. ${ }^{3}$ However, a limiting factor is that host stratification is a subjective judgment made by the treating surgeon as to what the patient's response to treatment will be. ${ }^{13}$

Judicious resection of necrotic tissue increases the chance of resolution of infection, but depending the anatomical type of chronic osteomyelitis, this surgical approach can potentially result in large defects or dead space..$^{3,10}$ Several strategies have been employed to manage this dead space including gentamycin beads, physiciandirected custom cement spacers, irrigation systems, antibioticloaded calcium sulphate pellets and, more recently, bioactive glass and gentamycin-impregnated collagen sponges. ${ }^{12,14}$

There is no treatment regimen that suits all patients, but current literature supports single-stage treatment strategies that incorporate a form of active dead space management. ${ }^{15}$ These single- 
stage strategies are driven in a large part by newer techniques of dead space management that do not require removal at a second sitting, such as polymethyl methacrylate (PMMA) spacers and gentamycin-impregnated beads, and success rates are comparable to traditional two-stage approaches. ${ }^{16}$

Empiric antibiotic regimens reported in the literature are varied, and the agents used should be tailored to local bacterial isolates and antibiogram patterns, and changed to pathogen-directed adjuvant therapy as soon as culture results are available. ${ }^{16}$

This study aims to present the outcome of management of chronic osteomyelitis of the appendicular skeleton according to an integrated approach at a dedicated bone infection unit.

\section{Methods}

A retrospective review of patient records was performed for the period from January 2016 to December 2018. During this time, all patients presenting to our unit with chronic osteomyelitis of the appendicular skeleton underwent treatment according to an integrated approach, demonstrated in Figure 1. This strategy represents our current standard of care for patients presenting with chronic osteomyelitis.

Exclusion criteria included patients younger than 12 years of age, infections of the spine and pelvis, and patients who did not attend at least one follow-up visit. Ethical approval as well as hospital board approval was obtained prior to data collection.

Chronic osteomyelitis was defined as: infection involving bone, with a duration of at least ten days, where the causative organisms were thought to have persisted either intracellularly or in interactive biofilm-based colonies. ${ }^{17,18}$

Pre-operative evaluation involved a thorough history, including main complaint, comorbid conditions, smoking, alcohol and substance abuse, previous surgical interventions and prior antibiotic therapy, as well as a comprehensive clinical examination. Clinical examination included evaluation of the local soft tissue quality, vascularity and neurological status of the affected limb.

Table I: Modified version of the original Cierny and Mader classification system that served to guide treatment strategy selection

\begin{tabular}{|c|c|}
\hline Classification & Characteristic \\
\hline $\begin{array}{l}\text { Physiological } \\
\text { Type A host } \\
\text { Type B host } \\
\text { Type C host }\end{array}$ & $\begin{array}{l}\text { No risk factors } \\
\text { Fewer than three minor risk factors } \\
\text { One major and/or three or more minor risk } \\
\text { factors }\end{array}$ \\
\hline $\begin{array}{l}\text { Pathoanatomy } \\
\text { I - Medullary } \\
\text { II - Cortical } \\
\text { III - Combined } \\
\text { (stable) } \\
\text { IV - Combined } \\
\text { (unstable) }\end{array}$ & $\begin{array}{l}\text { No cortical sequestration } \\
\text { Direct contiguous involvement in cortex only } \\
\text { Both cortex and medullary regions involved } \\
\text { As for III plus unstable prior to debridement }\end{array}$ \\
\hline $\begin{array}{l}\text { Nidus } \\
\text { Sequestrum } \\
\text { Implant } \\
\text { No identifiable nidus }\end{array}$ & $\begin{array}{l}\text { Cortical sequestrum present } \\
\text { Biofilm-based infection in the presence of implant } \\
\text { Minimal necrosis osteomyelitis }\end{array}$ \\
\hline $\begin{array}{l}\text { Impairment } \\
\text { Minimal } \\
\text { Severe }\end{array}$ & $\begin{array}{l}\text { Patient able to perform activities of daily living } \\
\text { Unable to perform activities of daily living }\end{array}$ \\
\hline \multicolumn{2}{|c|}{$\begin{array}{l}\text { (Reproduced, without any changes, under the terms of the Creative Commons } \\
\text { Attribution } 4.0 \text { International License from: Marais L, Ferreira N, Aldous C, Le Roux } \\
\text { T. The outcome of treatment of chronic osteomyelitis according to an integrated } \\
\text { approach. Strat Traum Limb Recon. } 2016 ; 11: 135-42 \text {. } \\
\text { [http://creativecommons.org/licenses/by/4.0/]) }\end{array}$} \\
\hline
\end{tabular}

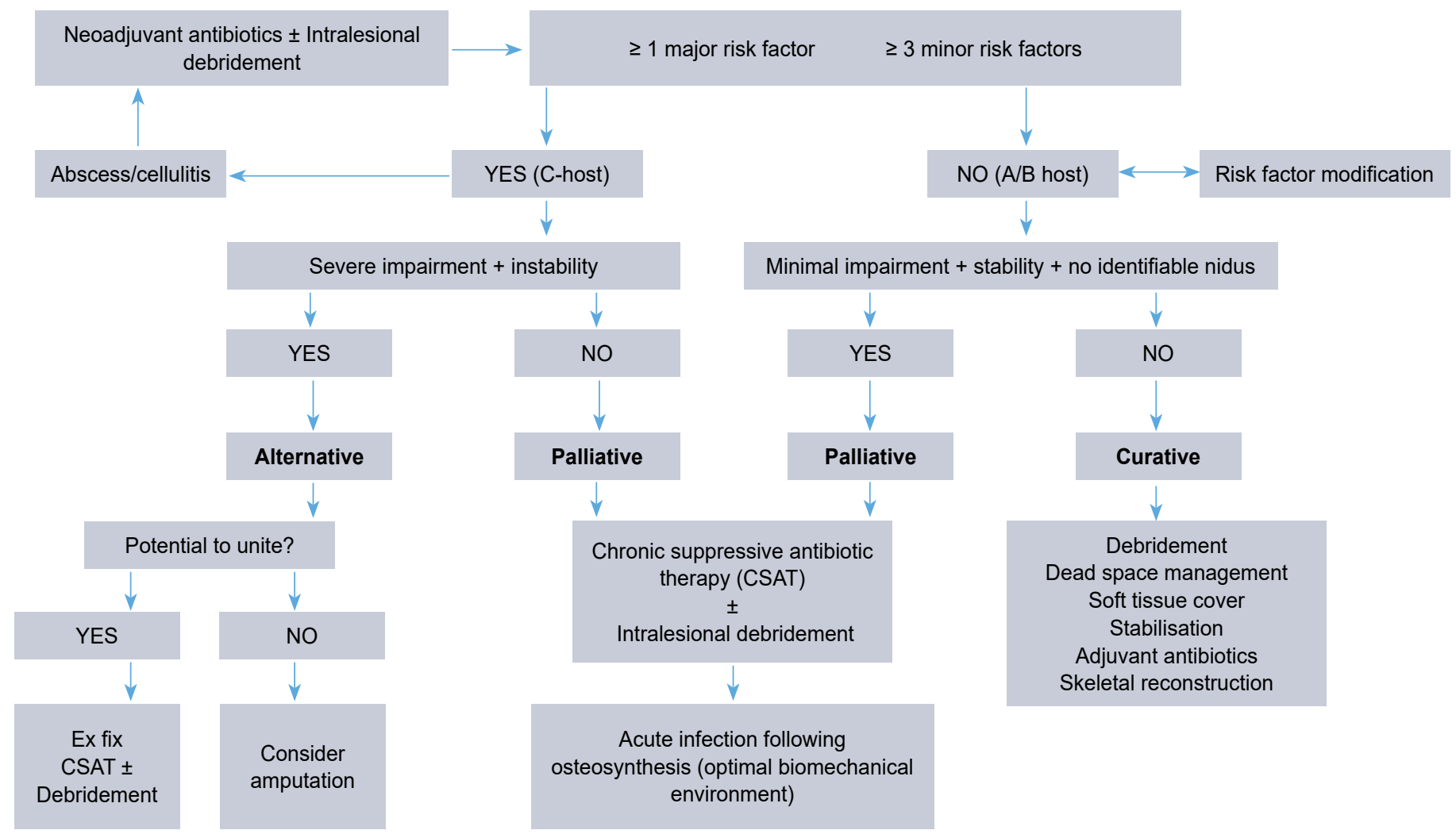

Figure 1. Treatment selection algorithm

(Reproduced, without any changes, under the terms of the Creative Commons Attribution 4.0 International License from: Marais L, Ferreira N, Aldous C, Le Roux T. The outcome of treatment of chronic osteomyelitis according to an integrated approach. Strat Traum Limb Recon. 2016;11:135-42.

[http://creativecommons.org/licenses/by/4.0/]) 
Table II: Risk factors used to stratify the physiological status of the host

\section{Major risk factors}

$\mathrm{CD}_{4}$ count $<350$ cells $/ \mathrm{min}^{3}$

Albumin $<30 \mathrm{~g} / \mathrm{l}$

$\mathrm{HbA} 1 \mathrm{C} \geq 8 \%$

Cellulitis or abscess formation

Malignancy at site of infection

Pathological fracture

\section{Minor systemic risk factors}

HIV infection

Anaemia

Smoking

Diabetes mellitus

Rheumatoid arthritis

Chronic lung disease

Chronic cardiac failure

Paraplegia/quadriplegia

Drug or substance abuse

Chronic corticosteroid use

Active tuberculosis

Ischaemic heart disease

Cerebrovascular disease

Compliance and motivation

Age $>65$ years
Minor local risk factors

Poor soft tissue requiring flap

Chronic venous insufficiency

Peripheral vascular disease

Previous radiation therapy

Surgery will result in instability

Adjacent joint stiffness/arthritis

Heterotopic ossification

Failed reconstruction elsewhere

Foot involvement

Pelvic involvement

Adjacent joint involvement

Segmental resection $\geq 60 \mathrm{~mm}$ required to achieve cure

(Reproduced, without any changes, under the terms of the Creative Commons Attribution 4.0 International License from: Marais L, Ferreira N, Aldous C, Le Roux T. The outcome of treatment of chronic osteomyelitis according to an integrated approach. Strat Traum Limb Recon. 2016;11:135-42.

[http://creativecommons.org/licenses/by/4.0/])

Plain radiographs were used to identify any sequestra or cloaca and assess skeletal stability. Computed tomography (CT) was predominantly used in type III and type IV cases where skeletal stability was in doubt and more anatomical detail was required for surgical planning. Anatomical classification of disease, according to the Cierny and Mader classification, was based on radiological findings. Laboratory investigations assisted in stratifying the patient's physiological status and comprised a full blood count, iron studies, renal and liver function tests and a nutritional profile. Routine infection markers included leukocyte count (WCC), erythrocyte sedimentation rate (ESR) and C-reactive protein (CRP) levels. In cases of specific chronic illnesses, supplementary tests included CD4 count and viral load for HIV infection, and $\mathrm{HbA} 1 \mathrm{C}$ in cases of diabetes mellitus. Host stratification into type A, $\mathrm{B}$ or $\mathrm{C}$ hosts was done according to the modification of the Cierny and Mader classification as proposed by Marias et al. set out in Table.$^{13}$ The major and minor risk factors are listed in Table $/ I{ }^{13}$

Definitive surgical management was preceded by host optimisation and governed by five general principles comprising: judicious debridement of necrotic tissue according to point-of-care testing, dead space management, adjuvant-directed antibiotic therapy, soft tissue coverage and bone reconstruction as required. Pointof-care testing refers to the surgeon's decision on bone viability considering the intra-operative assessment of bone colour, texture, timbre (sound) and capacity to bleed (paprika sign).

The management of Cierny and Mader type I osteomyelitis included removal of the intramedullary nail, indirect unroofing with debridement via reaming of the medullary canal and dead space management using a modified Lautenbach irrigation system. ${ }^{19}$ Debridement consists of over-reaming of the canal, starting with reamer size equal to the diameter of the removed nail. The reamer sizes are then sequentially increased until bony debris is obtained, and no more biofilm is returned on reaming extractions. Debridement of the interlocking screw holes with a high-speed burr is followed by irrigation of the canal with a pulse-lavage system. An improvised continuous irrigation system delivering 1 litre of isotonic saline containing $80 \mathrm{mg}$ gentamycin at a rate of $125 \mathrm{ml}$ per hour is used. Irrigation was allowed to run freely until the effluent was clear. No routine cultures of the effluent were done.

Type II and type III osteomyelitis were managed by direct unroofing using a high-speed burr followed by dead space management and soft tissue reconstruction as required. Dead space management consisted of soft tissue reconstruction with or without Garacol${ }^{\circledR}$ gentamycin-loaded fleece insertion (Innocoll Pharmaceuticals, Ireland) in type II osteomyelitis and BonAlive ${ }^{\circledR}$
$\mathrm{HO}$ : haematogenous osteomyelitis

FRI: fracture-related infection

gunshot fractures (included in FRI)

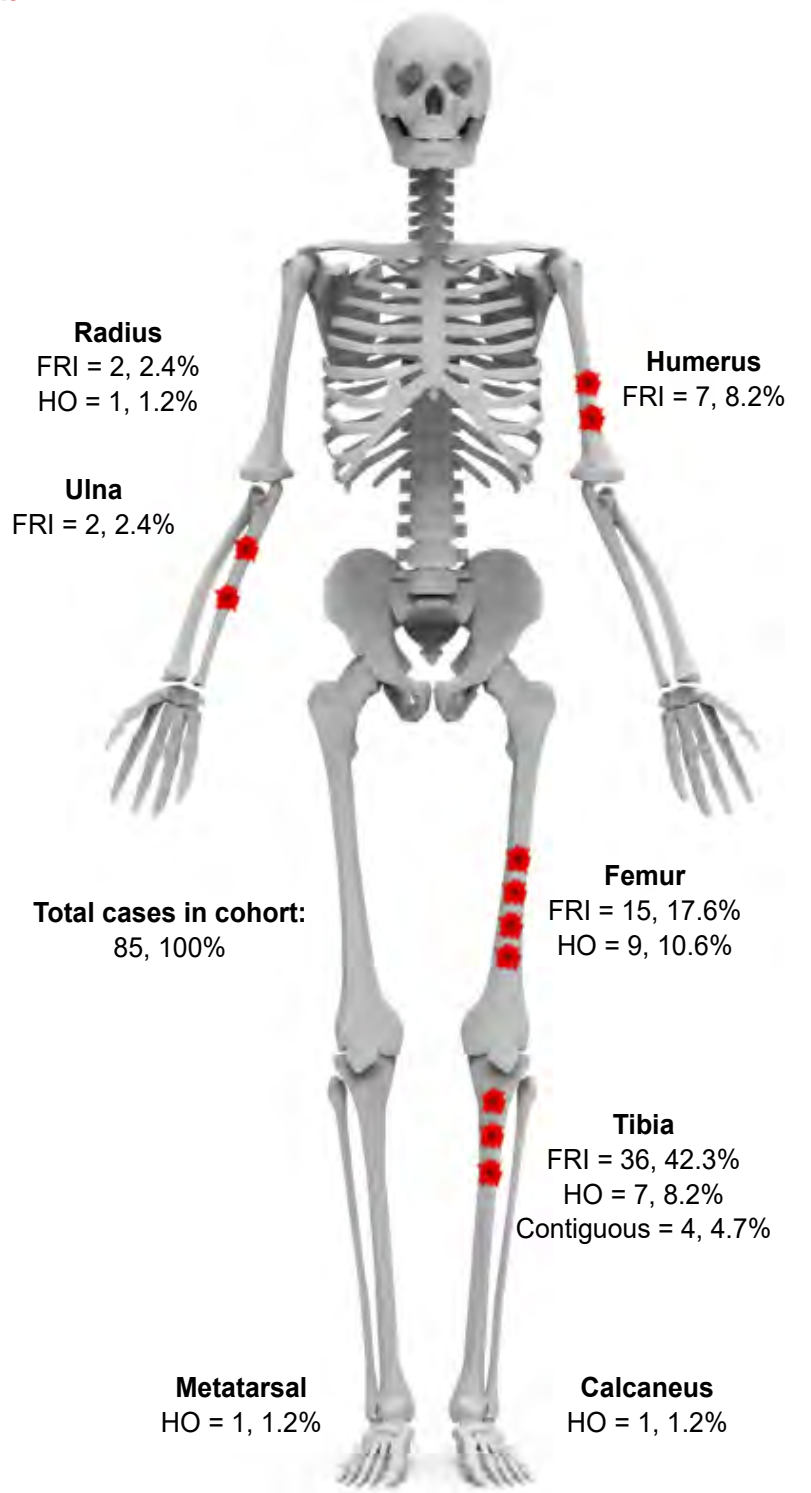

Figure 2. Distribution of anatomical sites of osteomyelitis 
bioactive glass (S53P4) (BonAlive Biomaterials Ltd, Turku, Finland) in all type III osteomyelitis cases. ${ }^{20}$

Most patients with diffuse osteomyelitis and skeletal instability (type IV) were managed by segmental resection of the necrotic bone followed by insertion of a physician-directed custom antibioticloaded cement spacer for dead space management. Palacos $R+G$ cement (Heraeus Medical), containing $0.5 \mathrm{~g}$ of gentamycin per $40 \mathrm{~g}$ pack, was used to construct the cement spacers. Once soft tissue reconstruction was achieved, bony reconstruction was considered. Bone defects smaller than $20 \mathrm{~mm}$ were treated by circular external fixation gradual shortening while larger defects were reconstructed by bone transport through an induced membrane using fine wire circular external fixation. ${ }^{12}$

Type C patients, irrespective of the anatomical grading, that had serious impairment of daily function, skeletal instability or active cellulitis were treated with an 'alternative strategy' including limited surgical interventions and chronic suppressive antibiotic therapy (CSAT). In cases where there was a reasonable chance of achieving union, limited debridement with external fixation was considered. In type C patients that had no realistic chance of

Table III: General and clinical characteristics of all patients

\begin{tabular}{|c|c|}
\hline & $n=80$ \\
\hline Age (years) & $36.25 \pm 13.39(80)$ \\
\hline Sex (\% male) & $74 \%(59)$ \\
\hline \multicolumn{2}{|l|}{ Original pathology } \\
\hline Fracture-related infection & $75 \%(60)$ \\
\hline Haematogenous infection & $21 \%(17)$ \\
\hline Contiguous infection & $4 \%(3)$ \\
\hline
\end{tabular}

Data is presented as mean \pm standard deviation for parametric data, median (interquartile range) for non-parametric data or frequencies, with the number of participants in parentheses. union, that would have an un-reconstructable bone or soft tissue defect post-debridement or would be left with a non-functional limb, amputation was considered.

\section{Results}

Between January 2016 and December 2018, 88 patients were treated for chronic osteomyelitis of the appendicular skeleton. Eight cases were excluded: one patient younger than 12 years of age, one pelvic infection, four patients who had simple soft tissue abscesses and two patients who were still waiting for definitive surgery. After exclusions, the final cohort consisted of 80 patients, comprising 59 men and 21 women with a mean age of 36.25 years (SD 13.39, range 12 to 67) (Table III). The involved anatomical sites are illustrated in Figure 2.

The follow-up for the cohort ranged from 1 to 29 months, with an average of 10.4 months. The case distribution according to the anatomical classification is shown in Table IV. Positive microbial cultures were obtained in $65(77 \%)$ cases (Table V). Bacterial culture information and sensitivity profiles were used to guide

Table IV: Case distribution according to Cierny and Mader anatomical classification

\begin{tabular}{|c|c|c|}
\hline COM anatomical type & Complications (n) & Recurrence (n) \\
\hline Type $1(n=21,26 \%)$ & $10 \%(2)$ & $10 \%(2)$ \\
\hline Type $2(n=14,18 \%)$ & $0 \%(0)$ & $7 \%(1)$ \\
\hline Type 3 $(n=24,30 \%)$ & $4 \%(1)$ & $4 \%(1)$ \\
\hline Type 4 $(n=21,26 \%)$ & $10 \%(2)$ & $5 \%(1)$ \\
\hline Total $(n=80,100 \%)$ & $6 \%(5)$ & $6 \%(5)$
\end{tabular}

Data is presented as frequencies with counts indicated in parentheses.

Table V: Bacterial isolates stratified according to Cierny and Mader anatomical type

\begin{tabular}{|c|c|c|c|c|c|}
\hline Culture information & C\&M I (n=21) & C\&M II (n=14) & C\&M III (n=24) & C\&M IV (n=21) & Total $(n=80)$ \\
\hline \multicolumn{6}{|c|}{ Organism cultured } \\
\hline No growth & $33 \%(7)$ & $14 \%(2)$ & $25 \%(6)$ & $10 \%(2)$ & $21 \%(17)$ \\
\hline Single organism & $29 \%(6)$ & $71 \%(10)$ & $67 \%(16)$ & $57 \%(12)$ & $55 \%(44)$ \\
\hline Multiple organisms & $38 \%(8)$ & $14 \%(2)$ & $8 \%(2)$ & $33 \%(7)$ & $24 \%(19)$ \\
\hline \multicolumn{6}{|c|}{ Gram-positive bacteria } \\
\hline Methicillin-sensitive Staphylococcus aureus & $24 \%(5)$ & $29 \%(4)$ & $40 \%(8)$ & $23 \%(6)$ & $28 \%(23)$ \\
\hline Methicillin-resistant Staphylococcus aureus & $10 \%(2)$ & $14 \%(2)$ & $25 \%(5)$ & $12 \%(3)$ & $15 \%(12)$ \\
\hline Enterococci & $14 \%(3)$ & $7 \%(1)$ & & & $5 \%(4)$ \\
\hline Enterobacter cloacae & & $14 \%(2)$ & & $12 \%(3)$ & $6 \%(5)$ \\
\hline Streptococci & $10 \%(2)$ & & $5 \%(1)$ & $4 \%(1)$ & $5 \%(4)$ \\
\hline Bifidobacterium & & & $5 \%(1)$ & & $1 \%(1)$ \\
\hline \multicolumn{6}{|c|}{ Gram-negative bacteria } \\
\hline Proteus species & $29 \%(6)$ & $29 \%(4)$ & & $19 \%(5)$ & $19 \%(15)$ \\
\hline Pseudomonas aeruginosa & $5 \%(1)$ & & $10 \%(2)$ & $19 \%(5)$ & $10 \%(8)$ \\
\hline Acinetobacter baumanni & & & & $4 \%(1)$ & $1 \%(1)$ \\
\hline Providencia stuartii & & & & $4 \%(1)$ & $1 \%(1)$ \\
\hline Klebsiella pneumoniae & & & $10 \%(2)$ & $4 \%(1)$ & $4 \%(3)$ \\
\hline Escherichia coli & & & $5 \%(1)$ & & $1 \%(1)$ \\
\hline Morganella morganii & $5 \%(1)$ & & & & $1 \%(1)$ \\
\hline Serratia marcescens & & $7 \%(1)$ & & & $1 \%(1)$ \\
\hline Citrobacter freundii & $5 \%(1)$ & & & & $1 \%(1)$ \\
\hline
\end{tabular}

Data is presented as frequencies with counts indicated in parentheses. 
antimicrobial therapy, but a full report on the antibiograms was beyond the scope of this article.

The overall complication rate for the cohort was $6 \%$ and included sterile drainage from the surgical site after management with bioactive glass (S53P4), refracture after hardware removal, infection of a bone graft site and development of non-union. Five patients experienced recurrence of infection after the initial procedure to eradicate infection, resulting in an overall resolution rate of $94 \%$.

\section{Type I}

Twenty-one patients (26\%) were treated for medullary osteomyelitis. All cases had prior insertion of an intramedullary nail. Treatment included removal of the intramedullary nail, indirect unroofing with intramedullary reaming and dead space management using a continuous irrigation system. Nine (43\%) patients did not complete six months follow-up, but all nine had complete resolution of symptoms at the last visit.

Complications were observed in two cases (10\%). One patient who initially received treatment for a septic cephalo-medullary femoral nail sustained a refracture four months after removal of the nail. The patient was deemed infection-free at the time of repeat nail insertion and no recurrence of infection was experienced 11 months after fracture fixation. A second patient who had a septic tibial nail removed was thought to have achieved union at the time of surgery, but at follow-up was found to have had a non-union. Union was achieved after circular external fixator application and the patient was infection-free at last follow-up, one year after the initial surgery.

Two patients (10\%) experienced recurrence of infection. The first defaulted antibiotic therapy during incarceration and was awaiting reoperation at the time of review. The second patient was a known smoker, who experienced recurrence of femoral osteomyelitis symptoms. He underwent a sequestrectomy five months later after which resolution of the symptoms was achieved.

\section{Type II}

Fourteen patients (18\%) were diagnosed with superficial osteomyelitis, including 11 (79\%) fracture-related infections, one $(7 \%)$ case of osteomyelitis of exposed bone following burn wounds, one case $(7 \%)$ of penetrating injury without an underlying fracture, and one case $(7 \%)$ of chronic osteomyelitis one year following haematogenous spread. All cases underwent singlestage debridement and soft tissue closure either by primary closure or flap. Seven (50\%) patients were lost to follow-up before six months.

One patient $(7 \%)$ had recurrence of infection. This case was a 26-year-old smoker and known substance abuser with osteomyelitis of the humerus after fracture fixation. He defaulted initial follow-up and returned four months later with an acute recurrence of infection.

\section{Type IIII}

Twenty-four patients (30\%) had localised osteomyelitis with a full thickness cortical sequestrum. Twelve cases $(50 \%)$ were due to fracture-related infections, $11(46 \%)$ were secondary to haematogenous spread and one (4\%) followed a severe burn that resulted in exposed bone. All were treated with judicious debridement and the use of bioactive glass (S53P4) as dead space management and either primary closure or flap. One patient $(4 \%)$ did not complete six months follow-up but was asymptomatic at last review.
One patient (4\%) experienced a period of persistent wound drainage after dead space management with bioactive glass which later resolved spontaneously. The patient was asymptomatic at 24 months follow-up.

One patient (4\%) with osteomyelitis of the humerus after fracture fixation experienced recurrence of symptoms after the initial procedure. He is known to be a smoker and a poorly controlled type II diabetic. This patient had eradication of infection following repeat debridement and dead space management with bioactive glass.

\section{Type IV}

Twenty-one patients (26\%) were diagnosed with diffuse osteomyelitis and skeletal instability, of which $17(81 \%)$ were secondary to fracture-related infections and four (19\%) were secondary to haematogenous spread.

Seven $(33 \%)$ patients were managed by segmental resection followed by insertion of a physician-directed custom gentamycinloaded cement spacer. Bone defect reconstruction was accomplished by frame-assisted gradual shortening in four cases, of which one patient had proximal tibial lengthening after the initial shortening, and three instances of bone transport through an induced membrane with routine bone grafting at the docking site.

One patient (5\%) was treated with debridement and soft tissue closure while in all-wire frame fixation for an open tibia fracture, one $(5 \%)$ was treated with debridement and frame-assisted ankle arthrodesis, and one patient (5\%) was treated with a cement nail.

Eleven patients (52\%) underwent limb ablation: four above-knee amputations, four below-knee amputations, one through-knee amputation, one above-elbow amputation and one ray amputation of the metatarsal. Five of these patients (46\%) were type $\mathrm{C}$ hosts that could not undergo extensive reconstruction. The rest were type $A$ or $B$ patients in which either an adjacent joint was also septic, the soft tissue defect was too extensive to reconstruct, or the patient requested ablation.

Two (10\%) patients in this group did not complete six months follow-up, but both these patients had complete resolution of symptoms at the time of the last visit.

Two patients (10\%) experienced complications. One, who had had a septic femoral nail removed in 18 years prior, subsequently defaulted CSAT and follow-up, and presented to our unit as a type $C$ host with skeletal instability, acute cellulitis and systemic sepsis. The patient passed away after initial treatment with debridement and Lautenbach irrigation followed by above-knee amputation. The second patient was a 52-year-old smoker who sustained an open tibia fracture with bone loss. He underwent bone transport through an induced membrane to reconstruct an 80 $\mathrm{mm}$ bone defect. Docking site sepsis and an adjacent knee flexion contracture brought about the patient's request for limb ablation.

One patient $(5 \%)$ experienced recurrence, a 12-year-old girl, who initially presented with acute staphylococcal septicaemia and osteomyelitis of the tibia. She underwent resections of necrotic bone and frame-assisted bone transport through an induced membrane. Stability was achieved, but the infection recurred 11 months later.

\section{Discussion}

The aim of this retrospective review was to evaluate the outcome of management of chronic osteomyelitis of the appendicular skeleton according to an integrated approach at a dedicated bone infection unit.

A2013 Cochrane review of four randomised control trials reported a combined remission rate of $79 \%$ at 12 months. ${ }^{21}$ More recently, the management of osteomyelitis in dedicated multidisciplinary 
bone infection units have been shown to produce better results illustrating the highly specialised nature of this work. ${ }^{22-24}$ Similar results were seen in our series with successful eradication of infection in all Cierny and Mader anatomical types in $94 \%$ of cases using a single-stage approach and a tailored dead space management strategy. Apart from obviating the need for second surgery, single-stage management of chronic osteomyelitis is also more cost-effective and has been shown to offer similar if not better clinical outcomes in resolution rates of infection. ${ }^{23,25}$ In a systematic review of single-stage management of osteomyelitis over a 20-year period, Pincher et al. described non-recurrence rates of $79 \%$ to $100 \%$ when all 'simple-debridement only' approaches were excluded. ${ }^{16}$ The authors concluded that not one dead space management technique was superior but that debridement without dead space management should be avoided. More specifically, Pincher et al. reported successful eradication of intramedullary infection in $83 \%$ of patients using the Lautenbach irrigation system, while a success rate of $96 \%$ was observed with the use of bioactive glass as dead space management following debridement. Our results support these findings, where success rates, for continuous irrigation and bioactive glass dead space management of $91 \%$ and $96 \%$, respectively, were observed.

Factors potentially contributing to the increased success rates include judicious debridement protocols, the availability of biodegradable antibiotic delivery systems as dead space management agents, improved sampling techniques intra-operatively, well-directed antibiotic therapies and the advances in prosthetic limb designs paving the way for a lower threshold of amputation surgery.

Failure to isolate an infecting organism was seen in 17 of the 80 patients $(21 \%)$ in our cohort. This is similar to the review by Pincher et al. in which $19 \%$ of the patients never had positive cultures. ${ }^{16}$

This review is limited by its retrospective nature and the relatively short mean follow-up time (ten months), compared to international literature (41.9 months) as well as the high rate of patients defaulting follow-up (5\%). ${ }^{16}$ In the South African setting poverty combined with large travel distances appears to affect general follow-up among research participants. Up to $21 \%$ of patients never returned for any post-operative visits in an observational study conducted on trauma patients in the Northern Cape. ${ }^{26}$ Similarly, a study conducted on patients receiving treatment for spinal tuberculosis, observed that $75 \%$ of patients were lost to follow-up within two years of diagnosis. ${ }^{27}$ It is, however, important to note that because of strict referral patterns within South Africa, should any patient return with complications, they will be referred back to the original treating facility.

\section{Conclusion}

The management of chronic osteomyelitis is complicated and resource-intensive. Using single-stage surgeries and tailored dead space management strategies according to a comprehensive integrated approach developed in South Africa, results comparable to international literature can be achieved.

\section{Ethics statement}

The authors declare that this submission is in accordance with the principles laid down by the Responsible Research Publication Position Statements as developed at the 2nd World Conference on Research Integrity in Singapore, 2010.

Ethics approval was obtained from the Stellenbosch University Human Research Ethics Committee N18/08/082 prior to commencement of data collection. All procedures were in accordance with the ethical standards of the responsible committee on human experimentation (institutional and national) and with the Helsinki Declaration of 1975 , as revised in 2008.

\section{Declaration}

The authors declare authorship of this article and that they have followed sound scientific research practice. This research is original and does not transgress plagiarism policies.

\section{Author contributions}

RGV: Data capture, data analysis, first draft preparation, manuscript preparation YST: Data capture, manuscript revision

JPG: Data capture, manuscript revision

NF: Study conceptualisation, data capture, data analysis, manuscript revision

\section{References}

1. Hall BB, Rosenblatt JE, Fitzgerald RH. Anaerobic septic arthritis and osteomyelitis. Orthop Clin North Am. 1984;15(3):505-16.

2. Haas DW, McAndrew MP. Bacterial osteomyelitis in adults: evolving considerations in diagnosis and treatment. Am J Med. 1996;101(5):550-61.

3. Cierny G. Surgical treatment of osteomyelitis. Plast Reconstr Surg. 2011;127(Suppl 1):190-204.

4. Gristina A. Biomaterial-centered infection: microbial adhesion versus tissue integration. Science. 1987;237(4822):1588-95.

5. Trampuz A, Zimmerli W. Diagnosis and treatment of infections associated with fracture-fixation devices. Injury. 2006;37 Suppl 2(2):S59-66.

6. Stewart PS. Multicellular resistance: biofilms. Trends Microbiol. 2001;9(5):204

7. Donlan RM. Biofilms: Microbial life on surfaces. Emerg Infect Dis. 2002;8(9):881-90.

8. Donlan RM, Costerton JW. Biofilms: survival mechanisms of clinically relevant microorganisms. Clin Microbiol Rev. 2002;15(2):167-93.

9. Walter G, Kemmerer M, Kappler C, Hoffmann R. Treatment algorithms for chronic osteomyelitis. Dtsch Aerzteblatt Online. 2012;109(14):257-64.

10. Simpson AHRW, Deakin M, Latham JM. Chronic osteomyelitis. J Bone Joint Surg Br. 2001;83-B(April):403-407.

11. Parsons B, Strauss E. Surgical management of chronic osteomyelitis. Am J Surg. 2004;188(1):57-66.

12. Marais LC, Ferreira N, Aldous C, Le Roux TLB. The management of chronic osteomyelitis : Part II - Principles of post-infective recontruction and antibiotic therapy. SA Orthop J. 2014;13(3):32-39.

13. Marais LC, Ferreira N, Aldous C, Le Roux TLB. The outcome of treatment of chronic osteomyelitis according to an integrated approach. Strateg Trauma Limb Reconstr. 2016;11(2):135-42.

14. Marais LC, Ferreira N, Aldous C, Le Roux TLB. The management of chronic osteomyelitis : Part I - Diagnostic work-up and surgical principles. SA Orthop J. 2014;13(2):42-48.

15. Metsemakers W-J, Fragomen AT, Moriarty TF, et al. Evidence-based recommendations for local antimicrobial strategies and dead space management in fracture-related infection. J Orthop Trauma. 2020;34(1):18-29.

16. Pincher B, Fenton C, Jeyapalan R, Barlow G, Sharma HK. A systematic review of the single-stage treatment of chronic osteomyelitis. J Orthop Surg Res. 2019;14(1):393

17. Lew DP, Waldvogel FA. Osteomyelitis. Lancet. 2004;364(9431):369-79.

18. Mader JT, Norden C, Nelson JD, Calandra GB. Evaluation of new anti-infective drugs for the treatment of osteomyelitis in adults. Infectious Diseases Society of America and the Food and Drug Administration. Clin Infect Dis. 1992;15(Suppl 1):S155-61.

19. Hashmi MA, Norman $P$, Saleh M. The management of chronic osteomyelitis using the Lautenbach method. J Bone Joint Surg Br. 2004;86-B(2):269-75.

20. Oosthuysen W, Venter R, Tanwar Y, Ferreira N. Bioactive glass as dead space management following debridement of type 3 chronic osteomyelitis. Int Orthop. 2020;44(3):421-28.

21. Conterno LO, Da Silva Filho CR. Antibiotics for treating chronic osteomyelitis in adults. Cochrane Database of Systematic Reviews. 2009;(3):CD004439.

22. Arias CA, Betancur MCT, Pinzon MA, et al. Differences in the clinical outcome of osteomyelitis by treating specialty: Orthopedics or infectology. PLoS One. 2015;10(12):1-14.

23. McNally MA, Ferguson JY, Lau ACK, et al. Single-stage treatment of chronic osteomyelitis with a new absorbable, gentamicin-loaded, calcium sulphate/ hydroxyapatite biocomposite: A prospective series of 100 cases. Bone $\mathrm{Jt} \mathrm{J}$. 2016;98-B(9):1289-96.

24. Jiang $N$, Zhao $X$, Wang $L$, et al. Single-stage debridement with implantation of antibiotic-loaded calcium sulphate in 34 cases of localized calcaneal osteomyelitis. Acta Orthop. 2020;3674:1-7.

25. Geurts J, Van Vugt T, Thijssen E, Arts JJ. Cost-effectiveness study of one-stage treatment of chronic osteomyelitis with bioactive glass S53P4. Materials (Basel). 2019;12(19):3209.

26. Badenhorst DHS, Van der Westhuizen CA, Britz E, Burger MC, Ferreira N. Lost to follow-up: Challenges to conducting orthopaedic research in South Africa. South African Med J. 2018;108(11):917-21.

27. Mann TN, Davis JH, Dyers R. Loss to follow-up among patients diagnosed with spinal tuberculosis at a tertiary hospital in Western Cape Province, South Africa: A retrospective cohort study. South African Med J. 2020;110(4):284-90. 